\title{
Buchstäblich fremd
}

Historische, politische und ästhetische Dimensionen

arabischer, japanischer und lateinischer Schrift

in interkultureller Literatur:

Frischmuth - Şenocak - Özdamar - Schami

Anne-Rose Meyer

\begin{abstract}
Writing is a central theme of intercultural literature. Above all, the Arabic script in its various forms, which are incomparable to the Latin script, is of interest to authors of German-language narrative works around the turn of the millennium, such as Rafik Schami, Zafer Şenocak, Barbara Frischmuth and Emine Sevgi Özdamar. This article shows that in their works, some of the aims of the visual and content-related theming of writing serves, are: to stimulate reflections on language and automated language use; to insert political and historical aspects of (language) politics into narratives, such as Ataturk's language reform in Turkey and its cultural effects; to include aesthetic aspects of writing as an ornamental work of art, which often escapes conscious attention when reading, and thus also to emphasize the material side of writing and printed works; to stage the activity of the laborious and sometimes impossible deciphering of a foreign script as a confrontation with family histories and larger historical contexts and thereby also to demonstrate the failure of cultural exchange. In this context, the work of Yoko Tawada is also of interest, who has dealt intensively with the history of the Latin alphabet and how it differs from Japanese writing
\end{abstract}

\section{Title}

Literally Alien. The Historical, Political and Aesthetic Dimensions of Arabic, Japanese and Latin Script in Intercultural Literature: Frischmuth-Şenocak-Özdamar-Schami

\section{Keywords}

Arabic script; Latin script; Japanese writing; Turkish history; language policies 


\section{Fremde Schriften in deutschsprachiger interkultureller Gegenwartsliteratur}

Bei Büchern, die für den deutschsprachigen Markt produziert werden, dürfen wir erwarten, dass sie auf Deutsch, und das heißt: im lateinischen Alphabet, geschrieben sind. Umso mehr stechen Werke ins Auge, auf deren Covern sich nichteuropäische Schriften finden lassen, z.B. Text auf Japanisch und Arabisch. Dies gilt etwa für Bücher der japanisch-deutschen Schriftstellerin Yoko Tawada, des aus Syrien stammenden Rafik Schami und der türkisch-deutschen Schriftstellerin Emine Sevgi Özdamar (vgl. Abb. 1-3).

\section{Abbildungen 1-3}
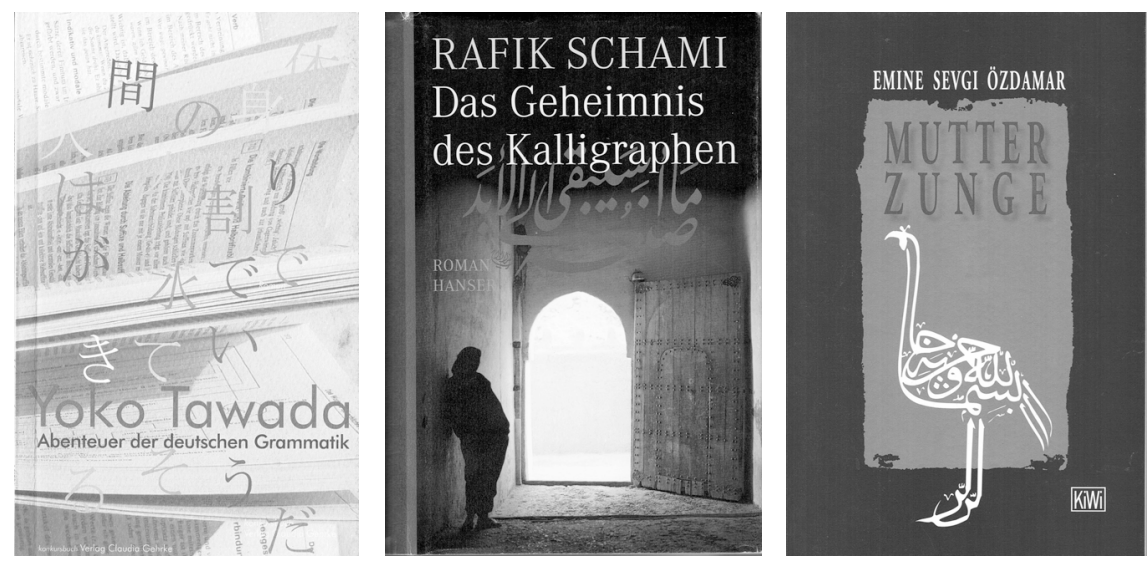

Valide Zahlen dazu, wie viele Menschen im deutschen Sprachraum des Japanischen und Arabischen mächtig sind, gibt es nicht. Es darf aber angenommen werden, dass die Verlage durch den Einsatz dieser Schriften an prominenter Stelle nicht auf die Erschließung neuer Käuferschichten zielen. Es leben derzeit in Düsseldorf, dem Zentrum für japanische Wirtschaftsaktivitäten in Deutschland, etwa 8400 Japaner. ${ }^{1}$ Das ist überschaubar und folglich mit Blick auf den deutschen Buchmarkt eher uninteressant. Arabisch wird heute - nach 2015 - von schätzungsweise einer Million Menschen in Deutschland beherrscht. ${ }^{2}$ Die abgebildeten Bücher von Schami und Özdamar wurden aber zu einer Zeit geschrieben, in der nur etwa 100.000 Menschen in Deutschland des Arabischen mächtig waren. Folglich zielen diese Peritexte nicht darauf, von einem größeren Lesepublikum im deutschsprachigen Raum verstanden zu werden. Worauf dann? Es ist ein auffälliges Merkmal interkultureller Gegenwartsliteratur, Fremdheit nicht nur auf inhaltlicher Ebene zu gestalten, sondern auch ästhetisch erfahrbar zu machen. Ein Mittel, das Lesepublikum zu befremden, es mit kulturell anderem $\mathrm{zu}$

1 Damit ist Düsseldorf »]apanstandort 1 in Kontinentaleuropa«, s. https://www.duesseldorf.de/inter nationales/partnerschaften/chiba/aktuelles/aktuelles-detailseite/newsdetail/wie-kam-so-viel-ja pan-nach-duesseldorf.html [Zugriff: 1.10.2021].

2 Vgl.https://www.destatis.de/DE/Themen/Cesellschaft-Umwelt/Bevoelkerung/Migration-Integra tion/Publikationen/Downloads-Migration/auslaend-bevoelkerung-2010200187004.pdf?__blob=pu blicationFile\&v=3 [Zugriff: 1.10.2021]. 
konfrontieren, ist, es buchstäblich an die Grenzen seines sprachlichen Verständnisses $\mathrm{zu}$ führen, indem Texte in einer anderen als der uns vertrauten lateinischen Schrift präsentiert werden. Dies ist in deutschsprachiger Gegenwartsliteratur, wie noch zu zeigen sein wird, hinsichtlich des Arabischen und des Japanischen immer auch verbunden mit historischen Reflexionen bzw. Evokationen vergangener Zeiten, vor allem - bzgl. des Arabischen - mit der Schriftreform Atatürks und Modernisierungsprozessen sowohl in der Türkei wie in arabischsprachigen Ländern und bzgl. der japanischen Schrift mit Ideen zu den Ursprüngen des lateinischen Alphabets.

\section{Arabische und lateinische Schrift in deutschsprachiger interkultureller Gegenwartsliteratur}

Betrachten wir literarische Werke näher, in denen es um arabische Schrift geht, lassen sich mehrere Formen der Darstellung ausmachen: Zafer Şenocaks Roman Gefährliche Verwandtschaft von 1998 etwa thematisiert zwar arabische Schrift und deren Geschichte mit Blick auf die Türkei in der Handlung, verzichtet jedoch gänzlich darauf, arabische Schrift im Buch zu visualisieren. Emine Sevgi Özdamars erste Buchveröffentlichung, Mutterzunge aus dem Jahr 1990 (vgl. Abb. 3), hat arabische Schrift auf dem Cover. In der Erzählung Großvater Zunge, der zweiten des Bandes, geht es zudem auf Handlungs- wie auf einer metafiktionalen Ebene um arabische Schrift. Sowohl auf dem Cover als auch auf dem Buchrücken von Barbara Frischmuths Roman Die Schrift des Freundes aus dem Jahr 1998 treten arabische Schriftzeichen gleichsam dreidimensional hervor (vgl. Abb. 4). In Rafik Schamis Roman Das Geheimnis des Kalligraphen dann, erschienen 2008, ist arabische Schrift optisch am präsentesten (vgl. Abb. 2). Auf Cover, Buchrücken, Vorsatz, Schmutztitel und den Seiten mit den Kapitelüberschriften finden wir arabische Schrift, genauer: Kalligraphien des syrischen Gegenwartskünstlers Ismat Amiralai. Diese enthalten die wörtliche Übertragung der deutschen Titel bzw. Überschriften ins Arabische. ${ }^{3}$ Auf Handlungsebene ist das Thema der arabischen Schrift bei Schami ebenfalls omnipräsent. Dies indiziert bereits der Titel, ähnlich wie auch bei Özdamar und Frischmuth.

Sowohl bei Özdamar als auch bei Frischmuth und Schami sind die Zeichen auf dem jeweiligen Cover Ergebnis einer sehr sorgfältigen Wahl. Diese zeigt sich bei Schami an der Zusammenarbeit mit eben dem erwähnten, in der arabischen Welt berühmten Ismat Amiralai sowie bei Schami, Özdamar und Frischmuth an der Wahl der verwendeten Schriftzeichen bzw. des verwendeten arabischen Textes. Diese erweitern die Interpretationsmöglichkeiten des deutschsprachigen literarischen Narrativs oder verstärken bestimmte, darin enthaltene Aussagen. Somit handelt es sich bei der Verwendung der arabischen Schrift in allen Romanen nicht allein um ein orientalistisch anmutendes, dekoratives Gestaltungselement, sondern um Zeichen, die in enger Verbindung mit den jeweiligen Inhalten stehen. Da der Einsatz arabischer Schrift aber auf die Peritexte beschränkt ist, zielt sie nicht darauf, optisch auf textinterne Mehrsprachigkeit hinzuweisen, so eine weitere Schlussfolgerung. Vergleicht man die genannten Werke, sind es eher sprachhistorische, -politische und soziolinguistische Aspekte, die dadurch fokussiert werden.

3 Diesen Hinweis verdanke ich Kiumars Aresumand-Gislandehi. 


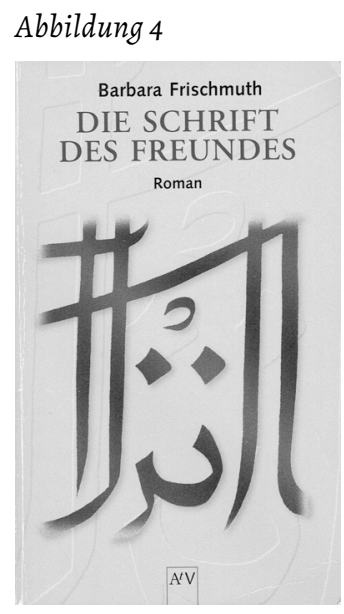

Ohne in die Details zu gehen, ist eine weitere auffällige Gemeinsamkeit der hier vorgestellten Werke, dass es inhaltliche Parallelen gibt. In allen Werken - auch in dem Roman Şenocaks, der keine optische Repräsentation des Arabischen bietet - werden Bezüge zur türkischen Geschichte hergestellt, und zwar zur Sprachreform Atatürks im Jahr 1928. ${ }^{4}$ Als weitere Gemeinsamkeit ist auszumachen, dass in allen Narrationen die Folgen von Schriftgebrauch und gewaltsamer politischer Veränderung von Schrift beispielhaft in ihren individuellen Auswirkungen auf Betroffene verdeutlicht werden. Als dritte Gemeinsamkeit ist zu bemerken, dass eine Eigenschaft des Arabischen immer wieder aufgegriffen wird, nämlich die, zwischen Bild und Text zu oszillieren. Darauf geben bereits die Cover erste Hinweise. In den Narrationen wird zudem immer wieder auch auf den der arabischen Schrift zugeschriebenen spirituellen Gehalt und die daraus entstehenden Spannungen zwischen Individuum und religiöser Gemeinschaft hingewiesen.

Mit der arabischen Schrift kontrastiert das lateinische Alphabet, denn alle Werke sind auf Deutsch geschrieben. Der ästhetische Eigencharakter der lateinischen Schrift liegt im Alltagsleben wie bei der literaturwissenschaftlichen Arbeit eines Native Speaker meist unterhalb der bewussten Wahrnehmungsschwelle. Der ästhetische Eigenwert der lateinischen Schrift tritt erst dann hervor, wenn dieser durch die Wahl bestimmter Drucktypen und eine graphisch auffällige Seitengestaltung betont wird. ${ }^{5}$ Man denke etwa an die von Stefan George höchstselbst besorgten Prachtbände seiner

4 Nach der Ausrufung der türkischen Republik 1923 erfassten die Reformbestrebungen unter der Ägide von Atatürk auch die Sprache: Nachdem die lateinische Schrift für die türkische Sprache in Staaten der UdSSR eingeführt worden war, sollte durch die Verwendung des lateinischen Alphabets auch in der Türkei der Kontakt der Turkvölker untereinander, auch grenzüberschreitend, gewahrt bleiben. Zudem bedeutete die Einführung der lateinischen Schrift symbolisch zugleich auch die Annäherung an den Westen und vergrößerte die Distanz zur osmanisch-islamischen Vergangenheit. Der Schriftwechsel unterstützte folglich auch die Säkularisierung der Türkei (vgl. Bayraktarlı 2009: 169-286).

5 Dieses Phänomen beschreibt Ulrike Franzki konzise: »Konnotative Bedeutungszuschreibung erfolgt in der Typographie grundsätzlich in einem Spannungsverhältnis von einfach codebezogener und komplexer interpretantenbezogener Rezeption - d.h. in einem Spannungsverhältnis von hochgradig konventionalisierten Semantisierungen, die in der Rezeption quasi automatisch aktualisiert werden 
Lyrik, an Konkrete Poesie oder visuelle Lyrik, an das Spiel mit Druckfarben, etwa bei Judith Schalansky, an Prosaexperimente aus den 1960er Jahren, die etwa Jürgen Becker in seinen Werken Felder, Ränder und Umgebungen angestellt hat.

\section{Zwischen Text und Bild: Schriften lesen und betrachten}

Bei den hier betrachteten Werken Schamis, Özdamars, Şenocaks und Frischmuths sind es die verschlungenen Formen arabischer Schrift, die - am auffälligsten bei Schami - die Geradheit und Gliederungsformen der lateinischen Schrift kontrastiv bemerkbar werden lassen. Und noch ein weiterer Punkt ist wichtig: Während wir als Lesende mit deutscher Muttersprache gar nicht anders können, als lateinische Schrift quasi automatisch und spontan zu entziffern, entzieht sich das gedruckte Arabische wohl dem Verständnis der meisten Mitteleuropäer. Für die Leserschaft von Schamis oder Özdamars oder Frischmuths Buch ist bedeutsam, dass es sich beim Arabischen um eine nichtindoeuropäische Sprache handelt, deren Schrift nicht in eine wenigstens teilweise identifizierbare Lautfolge übertragen und dadurch eventuell wenigstens partiell übersetzt werden kann. Der semiotische Referenzaspekt des Deutschen tritt bei dieser Art der optischen Präsentation in den Vordergrund, der des Arabischen nicht nur im übertragenen Sinn in den Hintergrund. Um es mit Aleida Assmann zu sagen, haben wir es mit einem »einfachen semiotische[n] Gesetz« zu tun, das die »inversive Relation von Anwesenheit und Abwesenheit (Assmann 1988: 238) markiert.

Wir können festhalten, dass Lesen »einerseits nie allein Entschlüsseln von Bedeutung« meinen kann, »da Texte immer auch materielle, visuelle, typografische [...] Momente enthalten« (Grizelj 2014: 64), die mehr oder weniger auffällig Wahrnehmung und Verständnis beeinflussen. Am auffälligsten ist dies wohl in der Werbung, wenn etwa durch schriftliche Rundungen beispielsweise die sahnig-cremige Konsistenz eines Eises evoziert werden soll. ${ }^{6}$ Andererseits

können umgekehrt nie diese Elemente die Bedeutungssuche völlig suspendieren. Lesen ist solchermaßen immer ein Multitasking. Es gibt keine kategoriale Trennung von visuellen und kognitiven, von materiellen und bedeutungshaften Elementen, also keine kategoriale Trennung von Buchstabenwert und Bild, von Diskursivität und Bildlichkeit, von Materialität und Referenzialität, von materieller Struktur und Zeichenstruktur. Zeichen, Texte, Sprachen, Schriften müssen gesehen und gelesen werden (Grizelj 2014: 64; Hervorh. i.O.).

Auch Susanne Wehde betont die Wichtigkeit von Typographie, Layout, Schrift als mediale und materielle Eigenschaften literarischer Texte gegenüber der »zeichentheoretisch naiven Entgegensetzung von Schrift- und Bildmedien« (Wehde 2000: 11, vgl. auch 90).

- und kontextabhängigen konnotativen Bedeutungszuschreibungen, die vom Rezipienten erst interpretativ hergeleitet werden.«(Franzki 2011: 149)

6 Vgl. hierzu auch die Ausführungen Franzkis (2011: 297-299) zu den Veränderungen der Schrift in der

Markenhistorie von Produkten der Marke Nivea, insbes. der Nivea-Creme. 
In den hier vorgestellten literarischen Texten selbst nun kommt arabische Schrift allenfalls auf dem Cover, aber nicht mehr gedruckt im Haupttext vor. Auch in der Erzählung Emine Sevgi Özdamars, in der man diverse Listen solcher arabischer Wörter findet, die auch heute noch das Türkische bereichern, sind diese in lateinischer Schrift wiedergegeben und damit wenigstens von ihrer Lautgestalt her für deutsche Leserinnen und Leser erkennbar. Zusätzlich wird die arabische Schrift beschrieben, das heißt bestimmte Charakteristika sind Teil der literarischen Narration. Darin kommt der arabischen Schrift eine funktionelle Vielfalt zu: Reflexionen über Sprache und einen automatisierten Sprachgebrauch anzuregen; historische Aspekte von (Sprach-)Politik in Narrationen einzufügen, wie etwa Atatürks Sprachreform in der Türkei und deren kulturelle Auswirkungen; die Tätigkeit des mühevollen und bisweilen unmöglichen Entzifferns einer fremden Schrift als Auseinandersetzung mit Familiengeschichten und größeren historischen Zusammenhängen zu inszenieren und dadurch auch das Scheitern kulturellen Austauschs vorzuführen; und schließlich ästhetische Aspekte von Schrift als ornamentalem Kunstwerk miteinzubeziehen, die bei der Lektüre häufig der bewussten Aufmerksamkeit entgehen, und so auch die materielle Seite von Schrift und Druckwerken zu betonen. Dieser letzte Punkt ist besonders wichtig für alle hier vorgestellten Erzähltexte, denn mit der ornamental anmutenden arabischen Schrift kommt die Verwobenheit von literaturtheoretischer Reflexion, Ästhetik und literarischem Schreiben besonders prägnant zum Ausdruck.

\section{Bezüge zwischen Kalligraphie und Schrift}

Wenn einer der führenden Bildtheoretiker der heutigen Zeit, Gottfried Boehm, den Begriff des Bildes strikt von dem der Sprache abzugrenzen versucht (vgl. Boehm 2007), dann geht er von einem mitteleuropäischen Sprachverständnis und dem lateinischen Alphabet aus. Die arabische Schrift hingegen ist als Kalligraphie bereits seit Jahrhunderten ein zentraler Bereich islamischer Kunst. Bedingt durch das Bilderverbot im Islam wird Schrift zu dem Ausdrucksmedium, das kein antagonistisches, sondern ein komplementäres Verhältnis von Schrift und Bild begründet. Dies wird optisch an dem stilisierten Vogel auf dem Titelcover von Emine Sevgi Özdamars erstem Erzählband ebenso deutlich wie erzählerisch in Rafik Schamis Roman Das Geheimnis des Kalligraphen. Darin ist an mehreren Stellen das Oszillieren der Schrift zwischen Text und Bild Thema. So schildert Schami den Eindruck eines Kunden, der sich in den 1950er Jahren über das Angebot eines damaszenischen Kalligraphen kundig macht und dem eine Arbeit präsentiert wird, wie folgt:

Es war ein Cemälde aus senkrechten schlanken Linien und schwungvollen Schlaufen sowie einer Menge Punkte, das als Segensspruch für den Präsidenten gedacht war. Aber mehr als Allah konnte er nicht entziffern.

»lch bin kein Fachmann«, sagte er, »und möchte Sie bitten, mir das Bild zu erklären.« Der Mitarbeiter war etwas verwundert, lächelte aber freundlich und fuhr mit dem Zeigefinger über die Glasscheibe, entlang der Buchstaben eines jeden Wortes, und plötzlich schlüpfte ein ganzer Satz aus dem Knäuel: Führer des Volkes, Oberst Schischakli, Gottes Hand ist mit dir. 
Nassri war erstaunt, wie einfach sich der Text lesen ließ, aber schon nach wenigen Minuten verschwamm er wieder vor seinen Augen. Was blieb, waren die einzelnen Wörter, Allah, Schischakli und Führer. Der Rest verschwand im Wald der goldenen Buchstaben. (Schami 2008: 166)

An anderer Stelle heißt es über die Präsentation einer weiteren Kalligraphie:

Einmal brachte er ein großes Buch mit, dessen Seiten mit für sie unverständlichen Ornamenten aus verschlungener Schrift gefüllt waren. Da und dort erkannte sie ein Wort, einen Buchstaben, aber das Canze blieb ein Geheimnis. Die Buchstaben bildeten einen eleganten Dschungel aus schwarzer Farbe und weißen Zwischenräumen. (Ebd.: 291)

Es ließen sich diverse weitere Textstellen anführen, in denen der Übergang von Lesen zum Betrachten, Text und Bild verunklart wird. Dies wird textintern einerseits als hohe Kunst valorisiert, etwa durch die Gestaltung von Kalligraphen und Schriftliebhabern als zentrale Figuren, andererseits auch kritisiert, etwa in der Figurenrede eines Ministers für Schule und Bildung, der die arabische Schrift gerade wegen deren Tendenz zum Ornamental-Bildhaften reformieren will:

Durch die Kalligraphie passiert Eigenartiges. Sie wurde erfunden, um die Schrift, die Zeichen der Sprache, auf Papier zu verehren, und doch vernichtet sie die Sprache, indem sie sie unleserlich macht. Die Schriftzeichen verlieren ihre Funktion als Gedanken überliefernde Zeichen und verwandeln sich in reine Dekorationselemente. (Ebd.: 444)

Die Form hat in der Kalligraphie Vorrang vor der Lesbarkeit. Auf inhaltlicher Ebene von Schamis Roman wird also eine Auffassung von Schrift verdeutlicht, die in diametralem Gegensatz steht zu der Schrift, die beim Druck des 458 Seiten langen, auf Deutsch geschriebenen Romans verwendet wurde. Diese nämlich stellt ihren ästhetischen Eigencharakter zurück. Sie ist im Gegensatz zur Kalligraphie leserfreundlich, da sie von ihren ikonischen Eigenschaften größtmöglich distanziert ist. Gleichwohl lässt sich die dauernde Thematisierung der arabischen Schrift und Kalligraphie im Roman als Einladung verstehen, auch über den Charakter des lateinischen Alphabets als Ausdrucksmedium für die deutsche Schriftsprache zu reflektieren. Wie vor allem Sybille Krämer gegen Aleida Assmann hervorgehoben hat, sind alle Schriften »Hybridisierung von Sprache und Bild« (Krämer 2003: 158). Diskursivität und Ikonizität sind auch im Deutschen eine produktive Einheit, deren Gebrauchswert im Roman ex negativo erschlossen wird. Dies ist ein Aspekt, der in allen genannten literarischen Werken bedeutsam ist. Und noch ein weiterer Punkt ist wichtig: Wie bereits angedeutet, ist die Geschichte des Arabischen stark politisch konnotiert. Dies wird an der Nähe der Schrift zum Bild bzw. an der Kalligraphie als der wichtigsten künstlerischen Ausdrucksform islamischer Glaubensgemeinschaften deutlich. Damit ist ein Punkt erreicht, der alle hier genannten Werke verbindet. 


\section{5. (Sprach-)Politik, Geschichte und Literatur}

In allen Erzählwerken bietet die Thematisierung des Arabischen einen Zugang zur Vergangenheit: In Frischmuths Roman Die Schrift des Freundes versucht der Protagonist, der Alevit Hikmet, über die Kunst der Kalligraphie dem osmanischen Türkisch, der Sprache seiner älteren Familienmitglieder, näherzukommen. Dadurch will er mehr über seine eigenen Ursprünge und die kulturellen Grundlagen seiner Herkunftskultur erfahren. Dieses rückwärtsgewandte biographische Projekt steht in auffälligem Kontrast zu der zukunftsorientierten Tätigkeit der weiblichen Hauptfigur als Computerspezialistin, mit der Hikmet im Romanverlauf eine Liebesbeziehung verbindet: Die junge Wienerin Anna Margotti ist Teil eines internationalen Teams, das in diversen Programmiersprachen Software für die Regierung entwickelt, u.a. auch für eine sichere länderübergreifende Kommunikation und für die Überwachung von Bürgerinnen und Bürgern. Mit Hikmets Verschwinden und der Suche Annas nach ihm verbindet Frischmuth beide Ebenen: Um überhaupt eine klarere Vorstellung des geliebten Mannes zu erhalten, setzt sich Anna mit dessen Sprache, Familie und Kultur auseinander und geht gleichzeitig in kritische Distanz zu ihrer eigenen beruflichen Tätigkeit, die mit Hikmets Verschwinden zusammenzuhängen scheint. Zudem fungiert sSchrift im Roman als handlungsorganisierende Zentralmetapher: Die sSchrift auf der Stirn bedeutet in islamischen Ländern das Schicksal und mittels dieser traditionsreichen übertragenen Bedeutung verknüpft die Autorin Frischmuth ihre zunächst weit auseinanderliegenden Handlungsfäden miteinander und motiviert die Liebesbeziehung zwischen Hikmet und Anna. ${ }^{7}$

In Özdamars Erzählung Großvater Zunge geht es gleichfalls um den Beziehungsverlust zu älteren Schriftformen. Die Protagonistin beherrscht nur modernes Türkisch und sucht - wie die Figur Hikmet bei Frischmuth - ebenfalls den Weg zurück in die Geschichte ihrer Kultur und ihrer Familie. Explizit artikuliert Özdamar die von Atatürk eingeleitete kulturelle Wende, deren augenfälligster Effekt der Wechsel vom arabischen zum lateinischen Alphabet war, als brutalen Eingriff und Verlusterfahrung. So heißt es zur Sprachgeschichte der Türkei aus Perspektive der autodiegetischen Erzählerin:

Ich habe zu Atatürk-Todestagen [...] geweint, aber er hätte die arabische Schrift nicht verbieten müssen. Dieses Verbot ist so, wie wenn die Hälfte von meinem Kopf abgeschnitten ist. Alle Namen von meiner Familie sind arabisch: Fatma, Mustafa, Ali, Samra. Gottseidank ich gehöre noch zu einer Generation, die mit vielen arabischen Wörtern aufgewachsen ist. Ich suchte arabische Wörter, die es noch in türkischer Sprache gibt. (Özdamar 2002: 29) ${ }^{8}$

Die bereits erwähnten Listen solcher arabischen Wörter prägen die Erzählung optisch wie inhaltlich. Clara Ervedosa (vgl. 2015: 65f.) hat richtigerweise darauf hingewiesen, dass die deutsche Sprache und Schrift in der Erzählung Özdamars die Verbindung zur

7 Auch diesen Hinweis verdanke ich Kiumars Aresumand-Gislandehi (vgl. Frischmuth 2000: 352). Das Verschwinden des Aleviten Hikmet löst eine Reihe privater, psychologischer, familiärer und politischer Verwicklungen aus.

8 Es folgen in der Erzählung diverse Listen solcher Wörter (vgl. Özdamar 2002: 29, 39, 41, 46). 
Familien- und Landesgeschichte nicht herstellen kann: »In der Fremdsprache haben Wörter keine Kindheit« (Özdamar 2002: 44), heißt es in Özdamars Erzählung. Doch auch der Weg über das Arabische zeigt sich als nicht gangbar. Ihr Lehrer erweist sich im Verlauf der Erzählung als sehr religiös und damit als inakzeptabel für die Schülerin. Die Suche nach der verlorenen Sprache und Schrift aber lässt sich im Gesamtkontext des Özdamar'schen Werkes als Initialzündung für das autobiographisch grundierte Erzählprojekt lesen, das dann in drei Romanen auf den ersten Erzählband Mutterzunge folgt - die Trilogie Sonne aufhalbem Weg.

Auch in Şenocaks Roman Gefährliche Verwandtschaft ist die Sprachgeschichte der Türkei ein wichtiges Thema und Mittel der Annäherung an die familiäre Vergangenheit des Erzählers: Dieser erbt von seinem Großvater Notizbücher mit Aufzeichnungen in arabischer und kyrillischer Schrift. Eigene Entzifferungsversuche bleiben erfolglos, ein professioneller Übersetzer übernimmt die Aufgabe. Die Ergebnisse von dessen Bemühungen bleiben der Leserschaft vorenthalten, der Roman endet - ähnlich wie Özdamars Erzählung - offen, mit dem Beginn einer neuerlichen Suchbewegung. Es heißt:

Ich sehnte mich danach, tiefere Schichten meiner selbst zu finden. Diese Tiefe war nur durch die Entdeckung meiner Herkunft zu erreichen. Ich wollte nicht mehr wurzellos sein, unverantwortlich für alles, was länger als zwanzig Jahre her war. Plötzlich erschien mir Großvater als das Geheimnis, das zwischen mir und meiner Herkunft stand. (Şenocak 1998: 118)

Schamis Roman ist von den hier vorgestellten Werken das politischste, insofern dieser Autor die gesellschaftlichen Folgen eines bestimmten Schriftgebrauchs und Schriftverständnisses benennt und ausführlich zum Thema macht. Die zentrale Figur des Romans ist der Kalligraph Hamid Farsi. Er plant eine Schriftreform. Dadurch sollen arabische Buchstaben präziser definiert und damit weniger verwechslungsanfällig werden. Zudem sollen durch die Entwicklung neuer Zeichen auch aktuelle fremdund fachsprachliche Ausdrücke ins Arabische integriert werden. Dieses soll dadurch Anschluss an die Moderne finden. Situiert in den 1950er und 1960er Jahren zeigt der Roman die Gegenbewegung der Gruppe der sogenannten Reinen, die in diesen Versuchen eine Missachtung der Religion, der Sprache des Korans sehen. Der Kalligraph scheitert mit seinen Reformversuchen, wie diverse Kollegen vor ihm. ${ }^{9}$ Auch bei Schami lässt sich - ähnlich wie bei Özdamar - in dieser thematischen Orientierung auf die politischen Dimensionen von Schrift eine Motivation sehen für das eigene Schreiben in der Fremdsprache und in einem zunächst fremden Schriftsystem - nämlich auf Deutsch. Und auch Frischmuth macht in ihrem Roman aufmerksam auf die Vereinnahmung des Arabischen durch radikal-islamistische Gruppierungen.

9 Darunter der auch heute noch verehrte, reale Ibn Muqla im 10. Jahrhundert, dem das Buch gewidmet ist. 


\section{Funktionen von arabischer Schrift in deutschsprachiger interkultureller Literatur der Gegenwart}

Die (hier nur skizzenhaft vorgenommene) Zusammenschau der vier komplexen Erzähltexte zeigt am Beispiel des Arabischen folglich auf mehreren Ebenen, dass Schrift in deutschsprachiger Gegenwartsliteratur eine doppelte Funktion hat. Diese besteht erstens in der recht augenfälligen, also optischen Inszenierung von Fremdheit. Zugleich beziehen Autorinnen und Autoren mit dem Einsatz und der Thematisierung der arabischen Schrift auf der Handlungsebene auch soziale und politische Realitäten in Geschichte und Gegenwart ein. Schrift erweist sich damit als Medium, dem sowohl in diachroner wie synchroner Perspektive bedeutende gesellschaftliche, religiöse und individualpsychologische Funktionen zukommen. Diese stehen in untrennbarer Verbindung mit der Ästhetik von Schrift, was besonders am Arabischen mit dessen Verbindung zur Kalligraphie deutlich wird. Unterschiede zwischen Text und Bild, Lesen und Betrachten werden verunklart.

Zentral ist in allen untersuchten Texten, dass der Eindruck der Fremdheit des Arabischen immer wieder auf die Vorstellung einer seigenen Sprache - des modernen Türkischen, des Deutschen - zurückgespiegelt wird und idealerweise eine starke Selbstreflexion des Lesenden über dessen eigenes Schriftsystem bewirkt. Damit ist die zweite Funktion des Einsatzes von nichtindoeuropäischer Schrift in deutschsprachiger Gegenwartsliteratur angedeutet, nämlich die spezifische Verwobenheit von literaturtheoretischer Reflexion und literarischer Produktion aufzuzeigen: Das Arabische erhöht die Poetizität des Geschriebenen, indem es von der unilingualen Norm abweicht und dadurch eine verfremdende und damit entautomatisierte Wahrnehmung des lateinischen Alphabets ermöglicht. Die Thematisierung von Schrift in der Literatur verbindet folglich Poetizität, Sprach- und Gesellschaftskritik sowie historische Reflexionen in einer Weise, die auch heute nicht nur für interkulturelles Verstehen und Verständnis relevant ist, sondern generell für eine Theorie von Literatur, die auch medienspezifische und linguistische Aspekte einschließt. Diese Verbindung von Literaturtheorie und Schrift wird noch einmal besonders am letzten, dem Japanischen gewidmeten Beispiel deutlich.

\section{Japanisch in deutschsprachiger Gegenwartsliteratur}

Yoko Tawada ist eine Schriftstellerin, die sowohl auf Deutsch als auch auf Japanisch schreibt. In auffälliger Weise setzt auch Tawada beide Sprachen bei der Covergestaltung (vgl. Abb. 1), aber auch vielfach in den Haupttexten ein, um auf die Vielschichtigkeit von Kultur aufmerksam zu machen und neue Sinnzusammenhänge zu generieren. Und auch Tawada nutzt dazu eine Sprache, die sich - ähnlich wie das Arabische

- im deutschsprachigen Raum dem Verständnis der allermeisten Leserinnen und Leser entzieht. Dies ist ein beabsichtigter Effekt, denn für Tawadas Werk sind Kontraste konstitutiv, mit denen sie die Fremdheit von Sprachen und Schriftsystemen betonen kann.

Damit folgt Tawada einer Spur, die bereits Roland Barthes (vgl. 1970) in seiner wegweisenden Schrift L'empire des signes 1970 aufgezeigt hat. In dieser Schrift erhebt Barthes nicht den Anspruch, japanische Kultur so, wie er sie in einer bestimmten his- 
torischen Situation als Mitteleuropäer erlebt, sauthentisch zu schildern oder gleichsam deren Essenz seiner Leserschaft nahezubringen; Barthes versucht bekanntlich vielmehr, mittels der Erfahrung von Differenz Grenzen unserer symbolischen Ordnungen zu identifizieren. Diesem Ansinnen ist der Umstand förderlich, es bei Japan mit einer Kultur zu tun haben, deren Schriftäußerungen sich dem mitteleuropäischen Denken entziehen. Japan, Japanisches erscheint als unzugänglicher Bereich, der aber gerade durch seine Rätselhaftigkeit zum Projektionsraum taugt. Indem Barthes Bilder japanischer Schriftzeichen in sein Werk einarbeitet, ermöglicht er es seinen Leserinnen und Lesern, jedenfalls den meisten von ihnen, die nämliche Erfahrung zu machen.

Yoko Tawada zeigt uns den umgekehrten Fall: Ihr erster auf Deutsch publizierter Text trägt den Titel Wo Europa anfängt. Und obgleich eine Reise Thema ist - nämlich von Tokio nach Moskau -, geht es nicht um geographische oder politische Grenzen, die Europa definieren. Für die Autorin werden vielmehr unterschiedliche kulturelle Räume durch einen Wechsel des Schriftsystems markiert. Damit "gestaltet« die Autorin »bereits den Anfang ihres Schreibens in der alphabetischen Schrift im Kontext einer historisch-theoretisch fundierten Sprach- und Schriftreflexion « und rückt »die kleinste Einheit der Schrift ins Zentrum der Aufmerksamkeit« - Buchstaben. »Ausgehend von einzelnen Zeichen soll der Text weniger >etwas mitteilen<, stattdessen wird Schreiben primär als Setzen und Verschieben von Zeichen gefasst, die einen materiellen Eigenwert besitzen« (Kilchmann 2012: 351). Die Materialität der Sprachzeichen wird zum bildspendenden Medium, zum Ausgangspunkt einer surrealistisch anmutenden Szene. So heißt es in Wo Europa anfängt:

Der Zug kam in Moskau an [...]. Dort stand ein hoher Turm [...]. Auf der Spitze des Turms saß der Feuervogel und spie flammende Buchstaben, M, O, S, K, A, U, und diese Buchstaben verwandelten sich: M wurde zu Mutter [...]. O wurde zu Omul und schwamm mit S-Seepferdchen. K wurde zu einer Kugel, einer Wasserkugel. $U$ hatte sich schon längst in ein Ungeheuer verwandelt, das mir vertraut vorkam.

\begin{abstract}
Aber was war mit A? A wurde zu einer fremden Frucht, die ich noch nie gegessen hatteeinem Apfel. [...] Warum darf ich nicht die fremde Frucht essen? Also biß ich in den Apfel hinein und schluckte sein saftiges Fleisch hinunter. In diesem Moment verschwanden die Mutter, der Omul, das Seepferdchen, die Kugel und das Ungeheuer vor meinen Augen. Es wurde still und kalt. [...] Ich bemerkte, daß ich mitten in Europa stand. (Tawada 1991: 87)
\end{abstract}

Esther Kilchmann kommentiert zutreffend, dass Tawada die »Aufmerksamkeit für die graphische Buchstabenform [...] mit Autoren wie Franz Kafka, E.T.A. Hoffmann und Ernst Jandl« teile, »auf die sie sich in ihren Texten auch explizit bezieht.« (Kilchmann 2012: 351) Am Ende dieser phantasievollen Buchstabenverfremdung in Wo Europa anfängt steht ein Befund, mit dem die Erzählung auch schließt: »Ich bemerkte, daß ich mitten in Europa stand." (Tawada 1991: 87) Mit dieser Feststellung hat Tawada eine für die deutschsprachige Literatur und Kulturtheorie wichtige Blickumkehr vorgenommen:

Erstens: Der bereits von Wilhelm von Humboldt formulierten Auffassung, die lateinische Schrift sei ein neutrales, gleichsam reines Darstellungsmedium (vgl. Hum- 
boldt 1963: 86f.), wird widersprochen. Legt ein Blick in die westliche Geschichte der Medientheorie doch offen, dass »die Marginalisierung gerade der aisthetischen Qualitäten und materiellen Faktoren dieses Schriftsystems ein medientheoretisches Leitmotiv« (Halawa/Sachs-Hombach 2015: 37) darstellt, so zeigt die Perspektive der aus Asien kommenden Erzählerinstanz in Tawadas Narration eine entautomatisierte Wahrnehmung der lateinischen Schrift.

Zweitens: Europa, um dessen Grenzen - geographische, politische - gerade heutzutage wieder vermehrt und erbittert gestritten wird und als dessen Außenposten nicht jeder Politiker die russische Hauptstadt Moskau akzeptieren würde, erweist sich aus außereuropäischer Perspektive als Gebilde mit anderen Konturen als aus innereuropäischer. Indirekt erinnert Tawada an gemeinsame Wurzeln, nämlich an die Entstehung der kyrillischen Schrift aus dem griechischen Alphabet, aus dessen westlicher Variante sich dann bekanntlich das altitalische und später das lateinische Alphabet bildeten (vgl. Hussmann 1977: 11, 28). Davon unterscheiden sich Entstehung und Geschichte anderer Systeme - das der japanischen Schrift als Ausdrucksmedium einer nichtindoeuropäischen Sprache etwa - fundamental (vgl. ebd.: 27). Aus der Perspektive der aus Asien kommenden Erzählinstanz beginnt Europa nicht nur in Moskau, sondern hat geschichtliche (Sprach-)Wurzeln, die tiefer reichen, als wir im Alltag zu sehen gewohnt sind.

Drittens deutet Tawadas Schreibverfahren, aus einzelnen Buchstaben neue, phantastische Sinnzusammenhänge zu generieren, auf sehr alte Auffassungen auch von deutschen Buch-Staben hin - »verweist das aus dem Althochdeutschen überkommene Wort doch auf einen Holzstab mit Runenzeichen und die >Rune wiederum auf das Geheimnis, bzw. den Zauber.« (Kilchmann 2012:364)

\section{Ausblick}

Die Beschäftigung mit außereuropäischen Schriftsystemen wie dem Arabischen und dem Japanischen in deutschsprachiger Gegenwartsliteratur verdeutlicht sprachpolitische und historische Aspekte, die bei der Verwendung unseres Alphabets im Alltag unberücksichtigt bleiben, denn: "Schriften vollziehen demnach nicht alleine eine Übertragung vom Akustischen ins Visuelle; vielmehr vermögen sie aufgrund ihrer besonderen Ikonizität Bedeutungswelten zu stiften, die ohne ihre Konstitutionsmacht nicht existierten.«(Halawa/Sachs-Hombach 2015: 38) Dies wird anhand unvertrauter Schriftsysteme in besonderer Weise anschaulich. Die auch heute noch verbreitete Ansicht, bei der lateinischen Alphabetschrift handele es sich um ein ästhetisch neutrales Ausdrucksmedium, wird durch alle der eben genannten Autoren und Autorinnen Frischmuth, Schami, Şenocak, Özdamar, Tawada - durch einen verfremdenden Blick und verfremdende Schreibweisen widerlegt.

Es wäre weiter zu fragen, ob sich bei der Thematisierung außereuropäischer Schriftsysteme in der Literatur systematische Gemeinsamkeiten oder auch signifikante Unterschiede finden lassen. Ferner wäre der Einsatz nichtindoeuropäischer Schriften auch in diachroner Hinsicht zu beleuchten, etwa mit Blick auf - um ein prominentes Beispiel zu nennen - Annette von Droste-Hülshoffs Judenbuche oder romantische Chiffrenschriften. Zu fragen wäre mit Blick auf deutschsprachige Literatur auch nach dem Wechsel von Fraktur zu Antiqua und dessen Bedeutungen. Die latei- 
nischen Buchstaben jedenfalls, in denen die deutschsprachige Gegenwartsliteratur meist verfasst ist, halten noch viel Untersuchungsmaterial für uns bereit.

\section{Literatur}

Assmann, Aleida (1988): Die Sprache der Dinge. Der lange Blick und die wilde Semiose. In: Hans Ulrich Gumbrecht/Karl Ludwig Pfeiffer (Hg.): Materialität der Kommunikation. Frankfurt a.M., S. 237-251.

Barthes, Roland (1970): L'Empire des signes. Paris.

Bayraktarlı, Ishan Yılmaz (2009): Die Türkei im Umbruch: Schrift und Sprache als nationalistisches Politikum in der türkischen Revolution. Freiburg i.Br.

Boehm, Gottfried (2007): Wie Bilder Sinn erzeugen. Die Macht des Zeigens. Berlin.

Ervedosa, Clara (2015): »Auch die Wörter werden zu Körpern«. Body, Sexuality and Carnavalesque Writing in Emine S. Ozdamar's Stories Mutterzunge and Großvaterzunge. In: Zeitschrift für interkulturelle Germanistik 6, H. 1, S. 51-70.

Franzki, Ulrike (2011): Identität durch Schrift. Schrift durch Identität. Zum Kommunikationspotenzial von Schrift. Boizenburg.

Frischmuth, Barbara (2000): Nachbemerkung. In: Dies.: Die Schrift des Freundes. Roman. Berlin, S. 349-352.

Grizelj, Mario (2014): Leere Seiten `lesen`?! Über Jürgen Beckers Ränder. In: Anne-Rose Meyer-Eisenhut/Burkhard Meyer-Sickendiek (Hg.): Fluxus und/ als Literatur. Zum Werk Jürgen Beckers. München, S. 47-67.

Halawa, Mark A./Sachs-Hombach, Klaus (2015): Zur Medialität der Handschrift. In: Urs Büttner u.a. (Hg.): Diesseits des Virtuellen. Handschrift im 20. und 21. Jahrhundert. Paderborn, S. 33-45.

Humboldt, Wilhelm von (1963): Ueber die Buchstabenschrift und ihren Zusammenhang mit dem Sprachbau [1824]. In: Ders.: Schriften zur Sprachphilosophie. Werke in fünf Bänden. Bd. III. Hg. v. Andreas Flitner u. Klaus Giel. Darmstadt, S. 82-112.

Hussmann, Heinrich (1977): Über die Schrift. Wiesbaden.

Kilchmann, Esther (2012): Verwandlungen des ABCs. Yoko Tawada und die Kulturgeschichte des abendländischen Buchstabens. In: Yoko Tawada: Fremde Wasser. Vorlesungen und wissenschaftliche Beiträge. Hg. v. Ortrud Gutjahr. Tübingen, S. 350-367.

Krämer, Sybille (2003): >Schriftbildlichkeit oder: Über eine (fast) vergessene Dimension der Schrift. In: Dies./Horst Bredekamp (Hg.): Bild - Schrift - Zahl. München, S. 157-176.

Özdamar, Emine Sevgi ( $\left.{ }^{2} 2002\right):$ Großvater Zunge. In: Dies.: Mutterzunge. Erzählungen [1990]. Köln, S. 15-48.

Schami, Rafik (2008): Das Geheimnis des Kalligraphen. Roman. München.

Şenocak, Zafer (1998): Gefährliche Verwandtschaft. Roman. München 1998.

Tawada, Yoko (1991): Wo Europa anfängt. Tübingen.

Wehde, Susanne (2000): Typographische Kultur. Eine zeichentheoretische und kulturgeschichtliche Studie zur Typographie und ihrer Entwicklung. Tübingen. 
